\title{
Evaluating Projects in a Dynamic Economy: Some New Envelope Results
}

\author{
by \\ Chuan-Zhong Li* and Karl-Gustaf Löfgren*** \\ *Department of Economics, Uppsala University, and Department of Economics and \\ Society, Dalarna University, Sweden \\ **Department of Economics, Umeå University, S-901 87 Umeå, Sweden
}

\begin{abstract}
This paper is concerned with the modern theory of social cost-benefit analysis in a dynamic economy. The theory emphasizes the role of a comprehensive, forwardlooking, dynamic welfare index within the period of the project rather than that of a project's long-term consequences. However, what constitutes such a welfare index remains controversial in the recent literature. In this paper, we attempt to shed light on the issue by deriving three equivalent cost-benefit rules for evaluating a small project. In particular, we show that the direct change in net national product (NNP) qualifies as a convenient welfare index without involving any other induced side effects. The project evaluation criterion thus becomes the present discounted value of the direct changes in NNP over the project period. We also illustrate the application of this theory in a few stylized examples.

Key words: dynamic cost-benefit analysis, net national product, project evaluation, welfare index.

\footnotetext{
${ }^{*}$ The authors are grateful to the Swedish Research Council Formas for financial support and to Martin L. Weitzman, Geir B. Asheim, Thomas Aronsson, and two referees for very helpful comments. The usual disclaimer applies. Corresponding author: Karl-Gustaf Löfgren, Email: KarlGustaf.Lofgren@econ.umu.se
} 


\section{Introduction}

In the economics literature, it is well-known that the criterion function for socialcost benefit analysis of investment projects should be the present discounted value of future social profits (cf. Dasgupta et al., 1972; Little and Mirrlees, 1974; Drèze and Stern, 1987). However, the exact constituents of social profits depend largely on the characteristics of the particular project under consideration. While earlier studies from the 1970s deal mainly with the "second-best" constraints (with disequilibrium), the recent literature is directed more towards environmental problems related to the concept of sustainable development ${ }^{1}$. As most environmental issues, such as global climate change, biodiversity loss, and nuclear waste disposal, are likely to have a long-run impact on human welfare, dynamic models with an infinite time horizon are often used as a theoretical basis.

The change in emphasis from the "second-best" constraints to environmental concerns has also led to a paradigm shift in theorizing social cost-benefit rules. Instead of valuing a project's lifetime consequences, the modern theory of dynamic social costbenefit analysis places greater emphasis on the role of a finite, forward-looking welfare index for project evaluations. Through the lens of accounting prices, the welfare index equipped with a component of net investment would be able to capture even starting from its "implementation" stage, the complete contribution of the project over the entire future. As such, the evaluation criterion becomes the present discounted value (PDV) of the welfare index numbers over the project period, thus removing the need to explicitly predict the cost-benefit consequences over the entire future. If a project leads to a larger PDV of the index numbers, it will be considered socially profitable from an efficiency point of view.

A different, but closely related problem is to measure sustainable development using the same type of dynamic welfare index. As long as the index numbers do not decline over time, the prevailing social well-being will be sustainable (cf. Weitzman, 2001; Li an Löfgren, 2006). In an abstract sense, dynamic social cost-benefit analysis and sustainability measurement deal with essentially the same problem, if the elapse of time in the latter case is regarded as a "project". Any increase in the index

\footnotetext{
${ }^{1}$ See Aronsson and Löfgren (1998; 1999); Asheim (2000), Dasgupta et al. (1995; 1997); Dasgupta and Mäler (2000); and Weitzman (2000; 2001).
} 
numbers, caused either by an intentionally implemented project or simply by the time momentum, would indicate a welfare improvement. However, when it comes to details, differences may emerge due to the nature of the "project" types. For sustainability measurement, the time "project" moves along a single direction only. On the other hand, in cost benefit analysis, an intentional project may involve a multi-dimensional change in the economy such as the introduction of direct policy reforms and public investments as well as their induced side effects. The problems associated with such multi-dimensional causes and effects may, thus, merit further exploration.

It is, today, well-known that the current-value Hamiltonian is a theoretically correct dynamic welfare index (Weitzman, 1976, 2003). However, how the concept should be operationalized within the framework of cost-benefit analysis has been the subject of controversy in the recent literature (cf. Asheim, 2000; Dasgupta et al., 1994, 1997; Dasgupta and Mäler, 2000; Weitzman, 2000). Firstly, should it be defined as a utilitybased, presumably nonlinear Hamiltonian function or as a linearized version in the form of a money-metric net national product.? Secondly, concerning its composition, what effects should count? If we consider the case of a small public investment project this may involve direct changes in consumption, investment and capital, as well as inducing some secondary, indirect effects on the economy as a whole. Which of these effects should we take into account in evaluating the project? Should we include all possible effects or just a subset of them, say the direct effects? Is it necessary to have a cost-of-holding capital term in the index?

The present paper attempts to clarify these issues by deriving three dynamic costbenefit rules that all produce the correct answer for evaluating small projects ${ }^{2}$. We use Ockham's razor ${ }^{3}$ to pick the most convenient rule, the one that retains the direct effects on NNP only. After presenting the model setup in section 2, we show in section 3 that it is correct to estimate the present value of all the direct and indirect effects on consumption over the infinite horizon. Thereafter, we show that another way to measure the change in welfare is to use the present value of the change in the net social profit ${ }^{4}$, including both the direct and indirect effects on consumption

\footnotetext{
${ }^{2}$ The choice of evaluating small projects motivates our use of differential techniques.

3 "Entities must not be multiplied beyond what is necessary".

${ }^{4}$ A term coined by Dixit et. al. (1980).
} 
and capital formation, evaluated over the period of the only. Lastly, the envelope properties enable us to get rid of all indirect effects, including the cost-of-holdingcapital term. The final evaluation criterion becomes, conveniently, the present value of the changes in NNP during the project period. We also illustrate the application of this theory using a few stylized examples in section 4 . Section 5 sums up the study.

\section{A Multi-Sector Conditional Optimal Growth Model}

In order to derive our dynamic cost-benefit rule in its most general form, we consider a multi-sector growth model with all possible consumption and investment goods taken into account. Let $\mathbf{C}=\left(C_{1}, C_{2}, \ldots, C_{m}\right)$ be a m-dimensional vector of consumption flows at a given time $t$, which is supposed to exhaust all possible goods and services that are relevant to social welfare or to the standard of living of a representative individual. In addition to the usual market commodities, environmental services such as forest amenities, biodiversity and ecosystem functions, in flow terms, are also considered to be part of the consumption vector. This means that the prices of these services are rental prices. The utilitarian measure of intertemporal welfare at time $t=0$ can be expressed as

$$
W_{0}=\int_{0}^{\infty} U(\mathbf{C}(t)) \exp (-\theta t) d t
$$

where $U(\mathbf{C})$ is a given concave, non-decreasing, instantaneous utility function with continuous second order derivatives defined for $\mathbf{C} \geq 0$, and $\theta$ is the utility rate of discount. Let $\mathbf{K}=\left(K_{1}, K_{2}, \ldots, K_{n}\right)$ be a vector of capital goods, which is assumed to contain all types of capital goods in the economy. Net investments are, by definition, the change in capital stocks, i.e. $I_{i}=\dot{K}_{i}, i=1,2, \ldots, n$, which in a vector form can be expressed as

$$
\mathbf{I}=\dot{\mathbf{K}} \text {, given } \mathbf{K}(0)=\mathbf{K}_{0}>0
$$

At each point in time $t$, consumption $\mathbf{C}(t)$ and investment $\mathbf{I}(t)$ are allocated within the $(m+n)$-dimensional attainable-possibility set $S(\mathbf{K}(t) ; \alpha)$, conditional on a collection of parameters (cf. Drèze and Stern, 1987), $\alpha$, such that

$$
(\mathbf{C}(t), \mathbf{I}(t)) \in S(\mathbf{K}(t) ; \alpha)
$$


which is assumed to be strictly convex. The parameters $\alpha$ may represent any premise that modifies the feasible set for consumption and investment allocations. This includes aspects such as a given property right regime, a given taxation system, or an inherent public infrastructure, which are normally not optimized in the economic system. Conditional on the parameters, the social planner is assumed to maximize the current-value Hamiltonian at each point in time $t$,

$$
H(t)=U(\mathbf{C}(t))+\mathbf{\Psi}(t) \mathbf{I}(t)
$$

with respect to $\{\mathbf{C}(t), \mathbf{I}(t)\}$ subject to (3), where $\mathbf{\Psi}(t)$ is the $n$-dimensional vector of the utility shadow prices (co-state variables) of capital satisfying the following equation of motion

$$
\dot{\Psi}=\theta \Psi-\left.\nabla H_{\mathbf{K}}\right|_{*(t)}
$$

where the notion $\left.\right|_{*(t)}$ means evaluation along the optimal trajectory at time $t$.

Note that the feasible set for the optimization problem $S(\mathbf{K}(t) ; \alpha)$ contains a collection of governance parameters in addition to the resource constraints. Thus, the optimal trajectory of consumption, investment and capital also depends on the parameters $\alpha$. In other words, the solution paths for these variables are conditional optimum paths. Let $\left\{\mathbf{C}^{*}(\alpha, t), \mathbf{I}^{*}(\alpha, t), \mathbf{K}^{*}(\alpha, t)\right\}$ denote the conditional optimum trajectory, then the maximized intertemporal welfare can be expressed as

$$
W_{0}^{*}(\alpha) \equiv \int_{0}^{\infty} U\left(\mathbf{C}^{*}(\alpha, t)\right) \exp (-\theta t) d t
$$

At each point in time $t$, the maximized current-value Hamiltonian is given by

$$
H^{*}(\alpha, t) \equiv U\left(\mathbf{C}^{*}(\alpha, t)\right)+\mathbf{\Psi}^{*}(\alpha, t) \mathbf{I}^{*}(\alpha, t)
$$

and the net national product $\left(\mathrm{NNP}^{5}\right)$, which is linear in prices, can be derived as

$$
Y^{*}(\alpha, t)=\mathbf{P}^{*}(\alpha, t) \mathbf{C}^{*}(\alpha, t)+\mathbf{Q}^{*}(\alpha, t) \mathbf{I}^{*}(\alpha, t)
$$

where $\mathbf{P}^{*}(\alpha, t)=\nabla U\left(\mathbf{C}^{*}(\alpha, t)\right) / \lambda(t)$ and $\mathbf{Q}^{*}(\alpha, t)=\mathbf{\Psi}(\alpha, t) / \lambda(t)$ are, respectively, the money price vectors of consumption and investment at time $t$, with $\lambda(t)$ as the corresponding marginal utility of income. This completes the background description required for deriving the dynamic cost-benefit rules.

\footnotetext{
${ }^{5}$ Since the consumption vector exhaust all for well-being relevant goods and services and the investment vector involves all types of productive capital stocks including environmental assets, the concept of NNP here is meant to be comprehensive NNP.
} 


\section{The dynamic cost-benefit rule for a small policy reform}

Now, we address the dynamic cost-benefit rules for evaluating a small project, say, a small policy reform. Given that the parameters $\alpha$ set a premise for the dynamic optimization problem, we are concerned about the welfare effect of a change in these parameters. Let us consider a policy reform with a small change, $d \alpha$, from reference level $\alpha_{0}$ during the period $[0, T]$. This may cause changes in consumption and investment both within the project and the post-project period. Without any loss of generality, let us consider a small public investment project. The aim of the costbenefit analysis is to evaluate whether or not the resulting change, over time, in the stream of consumption and investment values time is welfare improving.

According to the discounted utilitarian theory, the reform $d \alpha$ is socially profitable if it increases utility wealth as defined in (6), i.e. $d W_{0}^{*}(\alpha)=\partial W_{0}^{*}(\alpha) / \partial \alpha \cdot d \alpha>0$, evaluated at $\alpha=\alpha_{0}$. However, as utility wealth is not directly observable by the social planner, it will prove useful to find a monetary alternative that can provide the same ranking in project evaluations (cf Aronsson et al, 2004). Under certain regularity conditions concerning the value function in (6), the effect of a reform $d \alpha$ can be evaluated by invoking Leibniz's rule such that

$$
\begin{aligned}
d W_{0}^{*}(\alpha) & =\int_{0}^{\infty} \nabla U\left(\mathbf{C}^{*}(t)\right) \cdot d \mathbf{C}(t) \exp (-\theta t) d t \\
& =\int_{t}^{\infty} \lambda(t) \mathbf{P}^{*}(t) d \mathbf{C}(t) \exp (-\theta t) d t \\
& =\lambda(0) \int_{0}^{\infty} \mathbf{P}^{*}(t) d \mathbf{C}(t) \exp \left(-\int_{0}^{t} r(\tau) d \tau\right) d t
\end{aligned}
$$

where $d \mathbf{C}(t)=\partial \mathbf{C}(t) / \partial \alpha \cdot d \alpha$ denotes the total effect on the vector of consumption at time $t \geq 0$. The second equality is due to the first-order condition $\nabla U\left(\mathbf{C}^{*}(t)\right)=$ $\lambda(t) \mathbf{P}^{*}(t)$ from a dynamic competitive equilibrium (Dixit et al, 1980). The third equality follows from the Euler equation linking the pure rate of time preference $\theta$ to the money rate of discount $r(\tau)$, i.e. $\dot{\lambda}(\tau)=\lambda(\tau)(r(\tau)-\theta)$. The solution can be written as $\lambda(t) e^{-\theta t}=\lambda(0) \exp \left(-\int_{0}^{t} r(\tau) d \tau\right)$. Since $\lambda(0)>0$ is an arbitrary scale parameter, we can, without loss of generality, normalize it to unity, and propose the following proposition.

Proposition 1 The effect of a small policy reform, d $\alpha$, over a period $t \in[0, T]$, on the utility wealth $W_{0}^{*}(\alpha)$ is completely captured by the change in the present discounted 
value of future consumption i.e.

$$
d W_{0}^{*}(\alpha)=\int_{0}^{\infty} \mathbf{P}^{*}(t) d \mathbf{C}(t) \exp \left(-\int_{0}^{t} r(\tau) d \tau\right) d t
$$

Although equation (10) represents the theoretically correct criterion for social cost-benefit analysis of a small project, there are practical difficulties in its application. First, the effect on consumption at each point in time involves all "general equilibrium" effects on the whole economy; and, second, the social planner has to evaluate all such effects over an infinite time horizon. Consider a small hypothetical bridge project that requires both labor and timber inputs, and which would cause a direct change in consumption in terms of foregone leisure. Once constructed, the bridge would also generate transport services that would induce further changes in the economy. In addition, the timber exacted from the forest for the construction of the bridge would also affect the growth of the remaining trees as well as the forest amenities. This would, in turn, induce further changes in the rest of the economy. In practice, to predict all such effects over an infinite time horizon would be very difficult, if not impossible. In the quest of an operational version of the dynamic cost-benefit rule, we rewrite the optimal value function, in the same spirit as in Leonard (1987) and Caputo (1990), as

$$
\begin{aligned}
W_{0}^{*}(\alpha) & \equiv \int_{0}^{\infty} U\left(\mathbf{C}^{*}(\alpha, t), \alpha\right) e^{-\theta t} d t \\
& \equiv \int_{0}^{\infty}\left\{U\left(\mathbf{C}^{*}(\alpha, t), \alpha\right)+\mathbf{\Psi}^{*}(\alpha, t)\left[\mathbf{I}\left(\mathbf{C}^{*}(\alpha, t), \mathbf{K}^{*}(\alpha, t), \alpha, t\right)-\dot{\mathbf{K}}^{*}(\alpha, t)\right]\right\} e^{-\theta t} d t
\end{aligned}
$$

where $\Psi^{*}(\alpha, t)$ is the vector of utility shadow prices per unit of capital stocks at time $t$, conditional on $\alpha=\alpha_{0}$.

Note also that by allowing the parameter vector $\alpha$ to directly enter the utility function $U(\mathbf{C}(\alpha, s), \alpha)$, we can model a direct perturbation of consumption as a part of the reform $d \alpha$. To fix ideas, we may specify the utility function as $U=U(\mathbf{C}(\alpha, s)+$ $\boldsymbol{\delta}(\alpha))$ with $\boldsymbol{\delta}(\alpha)=0$ for the pre-project case at $\alpha=\alpha_{0}$. A direct effect of the reform, $d \alpha$, would be $\partial U(\mathbf{C}(\alpha, s), \alpha) / \partial \alpha=\partial U(\mathbf{C}(\alpha, s), \alpha) / \partial \mathbf{C} \cdot \partial \boldsymbol{\delta}(\alpha) / \partial \alpha$ and all other indirect effects on utility would be reflected through $\partial \mathbf{C}(\alpha, s) / \partial \alpha$. Presumably, the number of non-zero elements of $\partial \boldsymbol{\delta} / \partial \alpha$ for a small project is much fewer than the total dimension of the complete consumption vector. For the hypothetical bridge 
example mentioned above, the only direct effect on consumption is the reduction in leisure time, though the indirect effects may involve all possible general equilibrium adjustments triggered by the transport service of the new bridge. In certain cases, where the project does not involve a direct change in the consumption vector, we would have $\partial \boldsymbol{\delta} / \partial \alpha=0$.

By taking the total differential of the optimal value function in (11) with respect to $\alpha$, we can obtain

$$
d W_{0}^{*}(\alpha)=\int_{0}^{T}\left[\mathbf{P}^{*}(t) \Delta \mathbf{C}(t)+\mathbf{Q}^{*}(t) \Delta \mathbf{I}(t)+\mathbf{R}^{*}(t) \Delta \mathbf{K}(t)\right] \cdot \lambda(t) \cdot e^{-\theta t} d t
$$

where $\mathbf{Q}^{*}(t)=\mathbf{\Psi}^{*}(t) / \lambda(t)$ and $\mathbf{R}^{*}(t)=[\dot{\boldsymbol{\Psi}}(\alpha, t)-\theta \mathbf{\Psi}(\alpha, t)] / \lambda(t)$ are the money prices of investment and capital rentals, respectively, and the expressions $\Delta \mathbf{C}(t)=$ $\left(\partial \mathbf{C}^{*} / \partial \alpha+\partial \boldsymbol{\delta} / \partial \alpha\right) d \alpha, \Delta \mathbf{K}(t)=\partial \mathbf{K}(t) / \partial \alpha \cdot d \alpha$, and $\Delta \mathbf{I}(t)=(\partial \mathbf{I} / \partial \mathbf{C} \cdot \partial \mathbf{C} / \partial \alpha+$ $\partial \mathbf{I} / \partial \mathbf{K} \cdot \partial \mathbf{K} / \partial \alpha+\partial \mathbf{I} / \partial \alpha) d \alpha$ denote, respectively, the total change in consumption, capital and investment at time $t$ (See Appendix for more details). Note that the expression $\mathbf{P}^{*}(t) \Delta \mathbf{C}(t)+\mathbf{Q}^{*}(t) \Delta \mathbf{I}(t)+\mathbf{R}^{*}(t) \Delta \mathbf{K}(t)$ in (12) is the well-known version of social profit (Dixit et al., 1980). Now, by using the Euler equation $\dot{\lambda}(t)=\lambda(t)(r(t)-$ $\theta$ ), we can substitute the expression $\lambda(t) \exp (-\theta t)$ in (12) for $\lambda(0) \exp \left(-\int_{0}^{t} r(\tau) d \tau\right)$ or simply $\exp \left(-\int_{0}^{t} r(\tau) d \tau\right)$ with the initial marginal utility of income $\lambda(0)$ normalized to unity. This leads to the following widely accepted form of the dynamic cost-benefit rule (cf. Dasgupta 2001, Arrow et al., 2003).

Proposition 2 Consider a small policy reform, da, over a period [0,T], which would lead to changes in consumption, investment, and capital stocks within the project period by $\{\Delta \mathbf{C}(t), \Delta \mathbf{I}(t), \Delta \mathbf{K}(t)\}_{0}^{T}$. The project is socially profitable if the present discounted value of the resulting social profits is positive, i.e.

$$
\int_{0}^{T}\left\{\mathbf{P}^{*}(t) \Delta \mathbf{C}(t)+\mathbf{Q}^{*}(t) \Delta \mathbf{I}(t)+\mathbf{R}^{*}(t) \Delta \mathbf{K}(t)\right\} \exp \left(-\int_{0}^{t} r(\tau) d \tau\right) d t>0
$$

What is the relationship between the dynamic cost-benefit rule (13) and the cost benefit rule in (10) derived in Proposition 1? It can readily be shown that they are two sides of the same coin. The rationale behind this is that the second and the third 
terms in the integrand of (13), together with the discount factor, constitute an exact differential $\frac{d}{d t}\left(\mathbf{Q}^{*}(t) \Delta \mathbf{K}(t) \exp \left(-\int_{0}^{t} r(\tau) d \tau\right)\right)$ such that

$$
\int_{0}^{T}\left[\mathbf{Q}^{*}(t) \Delta \mathbf{I}(t)+\mathbf{R}^{*}(t) \Delta \mathbf{K}(t)\right] \exp \left[-\int_{0}^{t} r(\tau) d \tau\right] d s=\mathbf{Q}^{*}(T) \Delta \mathbf{K}(T) \exp \left[-\int_{0}^{T} r(\tau) d \tau\right]
$$

This expression ${ }^{6}$ corresponds to the optimal value change at the end of the project period (in present value terms) resulting from the reform in period $t \in[0, T]$, i.e., $d \hat{W}(\mathbf{K}(T))=\frac{\partial \hat{W}(\mathbf{K}(T))}{\partial \mathbf{K}(T)} d \mathbf{K}(T) \exp \left(-\int_{0}^{T} r(\tau) d \tau\right)$. This is the present discounted value of future changes in consumption given by ${ }^{7} \int_{T}^{\infty} \mathbf{P}^{*}(t) d \mathbf{C}(t) \exp \left(-\int_{0}^{t} r(\tau) d \tau\right) d t$. Now it can be seen that, while the integral of the first term in (13) measures the within-period welfare effects, the integral of the last two terms over the project period $t \in[0, T]$ measures the welfare effects over the whole post-project period from time $\mathrm{T}$ onwards. The sum of these effects becomes exactly the same as the expression in equation (10) in Proposition 1.

Next, by using the same first-order necessary conditions for the within-projectperiod $t \in[0, T], \partial U^{*} / \partial \mathbf{C}+\mathbf{\Psi}^{*} \partial \mathbf{I}^{*} / \partial \mathbf{C}=0$ and $\mathbf{S}^{*}=\dot{\Psi}^{*}-\theta \mathbf{\Psi}^{*}=-\partial H^{*} / \partial \mathbf{K}$, along the conditional optimal trajectory with a given $\alpha=\alpha_{0}$, we can simplify the Dixit et al's (1980) social profit expression in (13) to obtain our dynamic envelope result. After having canceled all the indirect effects on consumption, investment, and capital stocks, what is left in the integrand of (13) is the variation in the comprehensive net national product as anticipated by Dasgupta et al (1995) and Dasgupta and Mäler (1997). The simplified dynamic cost benefit rule from (13) can then be expressed as

Proposition 3 Consider a small policy reform, d $\alpha$, over the interval $[0, T]$, which would lead to a direct perturbation in consumption and investment with $d \tilde{\mathbf{C}}(t)=$ $\frac{\partial \boldsymbol{\delta}(\alpha, t)}{\partial \alpha} d \alpha$ and $d \tilde{\mathbf{I}}(t)=\frac{\partial \mathbf{I}(\cdot)}{\partial \alpha} d \alpha$, respectively. We measure the change in the present discounted value of NNP over the project period by

$$
\int_{0}^{T}\left\{\mathbf{P}^{*}(t) d \tilde{\mathbf{C}}(t)+\mathbf{Q}^{*}(t) d \tilde{\mathbf{I}}(t)\right\} \cdot \exp \left(-\int_{0}^{t} r(\tau) d \tau\right) d t
$$

\footnotetext{
${ }^{6}$ Note that $\Delta \mathbf{K}(t)=0$ at $t=0$, since the initial stocks $\mathbf{K}(0)$ are given.

${ }^{7}$ Perhaps the simplest way to se this is to equalize the cost-benefit rules in Proposition 1 and 2 such that $\int_{T}^{\infty} \mathbf{P}^{*}(t) d \mathbf{C}(t) \exp \left(-\int_{0}^{t} r(\tau) d \tau\right) d t=\int_{0}^{T}\left\{\mathbf{Q}^{*}(t) \Delta \mathbf{I}(t)+\mathbf{R}^{*}(t) \Delta \mathbf{K}(t)\right\} \exp \left(-\int_{0}^{t} r(\tau) d \tau d t\right.$.
} 
If this change is positive, the project is socially profitable ${ }^{8}$; if it is negative, the project is socially unprofitable.

This clear-cut, dynamic cost-benefit rule may greatly facilitate practical costbenefit analysis of small projects. This rule only requires information about the direct effect of the reform without any need to trace all the general equilibrium effects. In Dreze and Stern's (1987) terms, the dynamic cost-benefit rule stated in (15) is based on an evaluation of the reform parameters or instruments, and does not require the consequences of the reform or the general equilibrium effects. After having canceled out all indirect effects, what remains in the integrand of the integral in (15) is exactly the variation in (comprehensive) NNP caused by the reform! In addition, the social planner only needs to evaluate the effects within the project period, since the consumption effects over the post-project period have already been taken into account by the change in the value of investment within the project period.

Compared with Dasgupta and Mäler (2000) who claimed that the money NNP can only be used for evaluating an instant project with capital stock kept intact, our dynamic cost-benefit rule in (15) is more general. We have shown that the money NNP is a perfect instantaneous linear welfare index for evaluating a more realistic project. Rather than imposing that the capital must to be intact, we allow it to change over time as indicated in (13). The reason why the capital cost term vanishes in (15) is that the cost of holding capital is exactly offset by the benefit it would have generated. It is worth mentioning, however, that this is generally only true along a first-best-like, conditional optimum path. The underlying assumption is that households and firms would be optimizing agents within the given non-optimized premises, which the project is supposed to change. Otherwise, as shown by Aronsson et. al (1997), changes in stocks will appear in cost-benefit rules when the economy contains other market imperfections.

\footnotetext{
${ }^{8}$ In other words, a generalized version of Fisher's Separation Theorem holds (cf Aronsson and Löfgren, 1999). For cost benefit rules under externalities, see Johansson and Löfgren (1997) and Aronsson et al (1997, 1998).
} 


\section{Illustration and interpretations}

In this section, we illustrate our theory by means of a few stylized examples. The first example attempts to give a sense of what the direct, indirect, short and longrun effects could be, and how our envelope results would simplify the evaluation of the project. The model employed for this purpose is a modified Ramsey model with a non-diminishing rate of capital productivity, and our "project" is an exogenous change in the productivity parameter during a finite period of time. The second one is based the Brock (1977) growth model, where environmental quality enters the utility function. The final example is of an infrastructure investment, which includes more than one capital stock in the model.

\subsection{A simple Ramsey model}

To simplify the illustration, we take advantage of the Ramsey growth model equipped with a logarithm utility function and a linear production technology. The society's intertemporal welfare is expressed as $w_{0}=\int_{0}^{\infty} \ln (c(t)) \exp (-\theta t) d t$, and the stock dynamics by $\dot{k}(t)=\mu k(t)-c(t)$, with initial stock $k(0)=k_{0}>0$, and productivity level $\mu>0$. Conditional on a given $\mu$, the optimal time path for consumption, investment and capital can be derived, respectively, as $c^{*}(t)=\theta k_{0} e^{(\mu-\theta) t}, i^{*}(t)=$ $k_{0}(\mu-\theta) e^{(\mu-\theta) t}$ and $k^{*}(t)=k_{0} e^{(\mu-\theta) t}$, and their corresponding prices are $p^{*}(t)=$ $\frac{1}{\theta k_{0}} e^{-(\mu-\theta) t}, q^{*}(t)=\frac{1}{\theta k_{0}} e^{-(\mu-\theta) t}$, and $s^{*}(t)=-\frac{\mu}{\theta k_{0}} e^{-(\mu-\theta) t}$. Note that the consumption and investment prices are the same here in this one-sector model with a homogenous, all-purpose good.

Now, we introduce a small project, a shift in productivity with $d \mu>0$, for a finite period of time $[0, T]$. We want to "evaluate" it using the three propositions, although the welfare effect in this example is obviously positive. The aim with this exercise is to show how the various effects should be handled for a given evaluation criterion, and how the envelope theorem can facilitate the process.

The direct perturbations of the project, as compared to the usual path, are $d \tilde{\imath}(t)=$ $\frac{\partial \dot{k}(t)}{\partial \mu} d \mu=k^{*}(t) \cdot d \mu=k_{0} e^{(\mu-\theta) t} d \mu$ and $d \tilde{c}(t)=0$, for $t \in[0, T]$. To apply the envelope result from proposition 3 , it suffices to calculate the present discounted value of these 
direct changes over the project period, i.e.

$$
d w_{0}=\int_{0}^{T} q^{*}(t) \cdot \Delta \tilde{\imath}(t) e^{-\theta t} d t=\frac{d u}{\theta^{2}}\left(1-e^{-\theta T}\right)
$$

Suppose that $\mu=0.05, \theta=0.10$, and $d u=0.01$, and $T=10$. Then the welfare change from (16) becomes $d w_{0} \approx 0.63>0$, indicating, as expected, that the "project" is welfare improving.

Next, let us try to apply proposition 2 , a result based on the notation of net social profit. Here, we need to calculate the overall changes in consumption, investment and capital, caused by the project, including all possible effects. By solving for the alternative path based on the after-project productivity level $\mu^{\prime}=\mu+d u$, and comparing it with the original path conditional on the pre-project level $\mu$, we find that the changes: $\Delta c(t)=\theta k_{0}\left(e^{\Delta u \cdot t}-1\right) e^{(\mu-\theta) t}, \Delta i(t)=k_{0}\left[\left(\left(u^{\prime}-\theta\right) e^{\Delta u \cdot t}-(u-\theta)\right)\right] e^{(\mu-\theta) t}$, and $\Delta k(t)=k_{0}\left(e^{\Delta u \cdot t}-1\right) e^{(\mu-\theta) t}$, are valid for the reform period $t \in[0, T]$. For this part of the analysis, the differences after the project period are not needed. Using proposition 2, then, we have the PDV of net social profits over the reform period as

$$
\begin{aligned}
d w_{0} & =\int_{0}^{T}\left(p^{*}(t) \Delta c(t)+q^{*}(t) \Delta i(t)+s^{*}(t) \Delta k(t)\right) e^{-\theta t} d t \\
& =\int_{0}^{T}\left\{\left(1-\frac{\mu}{\theta}\right)\left(e^{(\Delta u-\theta) t}-e^{-\theta t}\right)+\frac{1}{\theta}\left[\left(u^{\prime}-\theta\right) e^{(\Delta u-\theta) t}-(u-\theta) e^{-\theta t}\right]\right\} d t \\
& =\frac{\Delta u}{\theta(\Delta u-\theta)}\left(e^{(\Delta u-\theta) T}-1\right)
\end{aligned}
$$

Inserting the same parameter values above, the expression from (17) gives a numerical value $d w_{0} \approx 0.66>0$, extremely close to that from (16), in spite of its roundaboutsness The deviation from (16) would vanish if the project $d u$ could be reduced so as to be infinitesimal.

Finally, let us illustrate the application of proposition 1. In order to do this, we also need to predict the overall change in consumption beyond the project period, i.e. $\Delta c(t)=\theta k_{0}\left(e^{\Delta u \cdot T}-1\right) e^{(\mu-\theta) t}$ for $t>T$. The present discounted value of the complete stream of changes in consumption is then

$$
\begin{aligned}
d w_{0} & =\int_{0}^{\infty} p^{*}(t) \cdot \Delta c(t) e^{-\theta t} d t \\
& =\int_{0}^{T}\left(e^{\Delta u \cdot t}-1\right) e^{-\theta t} d t+\int_{T}^{\infty}\left(e^{\Delta u \cdot T}-1\right) e^{-\theta t} d t
\end{aligned}
$$


which ends up with exactly the same solution as in (17). It can now be seen that all three propositions give, in principle, the same results although the envelope result from proposition 3 is much simpler. The major complication with proposition 1 is that it needs consumption data over an infinite horizon, and the difficulty associated with proposition 2 lies in its involvement of multiple effects in investment and capital.

\subsection{The Brock growth model}

Here we consider a modified Ramsey growth model with pollution (Brock, 1977) based on more general utility and production functions. The planner's objective is to maximize the present discounted value of future utilities i.e.

$$
\max w_{0}=\int_{0}^{\infty} u(c, x) \exp (-\theta t) d t
$$

subject to $\dot{k}=f(k, e)-c-g(\alpha)$ and $\dot{x}=e-\sigma(\alpha) x$ with $k(0)=k_{0}$ and $x(0)=x_{0}$ (cf. Aronsson et al,1997). Here $c$ denotes consumption and $x$ the stock of pollution. Both affect the instantaneous utility, $u(c, x)$, the variable $e$ represents the emission of pollutant and $k$ the physical capital stock. Both of these enter the production function. In the pre-project situation, the investment in cleaning technology, $g(\alpha)$, is assumed to be zero, and the assimilative capacity of the natural environment is a constant $\sigma\left(\alpha_{0}\right)$. Now, to evaluate a small investment project $d \alpha>0$ with $g^{\prime}(\alpha)>0$ and $\sigma^{\prime}(\alpha)>0$ for all $t \geq 0$, we can apply the dynamic cost-benefit rule in (15) such that, if the expression

$$
d w_{0}=\int_{0}^{\infty}\left[\mu(t) \sigma^{*}(t)-\lambda(t) g^{\prime}(\alpha)\right] \exp (-\theta t) d t=\int_{0}^{\infty} \frac{\partial H^{*}(t)}{\partial \alpha} d t>0
$$

then, the project is said to be socially profitable. Here $\partial H^{*} / \partial \alpha$ is the partial derivative of the present value Hamiltonian with respect to $\alpha$, evaluated along the optimal path, and $\mu(t)<0$ is the shadow price of the stock of emissions. In monetary terms, we may write this expression as

$$
\frac{d w_{0}}{\lambda_{0}}=\int_{0}^{\infty}\left[\bar{\mu}(t) \sigma^{\prime *}(t)-g^{\prime}(\alpha)\right] \exp \left[-\int_{0}^{t} r(\tau) d \tau\right] d t>0
$$


where $\bar{\mu}(t)=\mu(t) / \lambda(t)$ corresponds to the willingness-to-pay for a marginal reduction in the pollution stock. Note that only the direct or partial effects of the project are included here in the dynamic cost-benefit rule. This is because all other indirect or induced effects on consumption, investment, emission, and changes in the two types of capital stocks are canceled along the conditional ( $\left.\alpha=\alpha_{0}\right)$ optimal trajectory.

\subsection{Infrastructure investment}

In this final example, we consider a growth model in which infrastructure investment may have a direct effect on consumption when this is defined in a broader sense so as to include leisure and recreation. The intertemporal welfare is formulated as $w_{0}=$ $\int_{0}^{\infty} u(c, l, x) \exp (-\theta t) d t$, and the stock dynamics equation are $\dot{k}=f(k, z)-c-b(a)$ and $\dot{z}=a-\gamma z$, where $c$ is the usual consumption, $k$ represents capital and $z$ infrastructure, say, the road net. For notational ease, we assume that only capital and the road net enter the production function either without labor input or with a fixed amount of labor. Thus, an infrastructure investment $d a$ would directly affect leisure through $l=l(a), l^{\prime}(a)<0$ as well as the environmental service through $x=x(a), x^{\prime}(a)<0$. To build a new road or improve an old one with $d a(t)$ at each year requires resource $k$ and labor input.

The business-as-usual case is a fixed, non-optimized stock of the road net with $z=z_{0}$. Road maintenance simply compensates the depreciation such that $a_{0}=$ $r z_{0}$. Now, suppose that the government plans an active road investment project over a period of $T$ years. This would involve direct changes in consumption by $\left.d \tilde{c}(t)=[d \tilde{l}(t), d \tilde{e}(t)]=\left\{l_{a}[a(t)]\right), x_{a}[a(t)]\right\} d a(t)$ and changes in investments by $d \tilde{\imath}(t)=[d \dot{k}(t), d z(t)]=\left[-b^{\prime}(a) d a(t), d a(t)\right]$, at year $t, t \in[0, T]$. The direct change in comprehensive NNP at year $t$ thus becomes

$$
\Delta N N P(t)=p_{l}^{*}(t) d \tilde{l}(t)+p_{e}^{*}(t) d \tilde{e}(t)-q_{k}^{*}(t) b^{\prime}(a) d a(t)+q_{z}^{*}(t) d a(t)
$$

where the $p:$ s and the $q$ :s denote the consumption and investment prices for the corresponding goods. The project evaluation according to our proposition 3, would thus be

$$
d w_{0}=\int_{0}^{T} \Delta N N P(t) e^{-\theta t} d t
$$


For more on the technical details of the dynamic Envelope Theorem, see Seierstad (1982).

\section{Concluding remarks}

We started out with a string of questions. Most of them, we hope all, have been answered by Propositions 1-3. The overall answer is that a cost-benefit rule under a first-best-like setting can be formulated in many different ways. In Proposition 1 , we show that the present value of the change in consumption from time zero to infinity is one answer, albeit a little impractical. In Proposition 2, we show that it can be rewritten as the present value of social profit over the project period. The two propositions are different sides of the same coin. The social profit concept can be further simplified by making use of the fact that certain envelope properties (first order conditions) hold over the project period. The cost of holding capital term now disappears, since the cost of holding capital is exactly offset by the benefit it would have generated. After having canceled out all indirect effects, we are, in Proposition 3 , left with a linear index consisting of the present value of the direct effects on the comprehensive NNP (or the Hamiltonian) over the project period; i.e. whether it is a utility or money metrics does not matter. This is by far the most practical way of approaching project evaluation.

We have also provided a few stylized examples to illustrate our theory. It is demonstrated that the envelope results can greatly facilitate cost-benefit analysis of small projects, provided that the conditional optimum path assumptions hold. It is also worth mentioning that the main results presented in this paper can also be derived by starting from a much more general stochastic multi-sector growth model, using the envelope properties embedded in the Hamilton-Jacobi-Bellman equation. The deterministic Envelope Theorem and our proposition 3 would then surface as a special case.

\section{Appendix}

In this appendix, we show how to arrive at the "net social profit" notion of the costbenefit rule. By taking the total differential of the optimal value function in (11) with 
respect to $\alpha$, we obtain

$$
\begin{aligned}
d W_{0}^{*}(\alpha)= & \int_{0}^{\infty}\left\{\frac{\partial U^{*}}{\partial \mathbf{C}}\left[\frac{\partial \mathbf{C}^{*}}{\partial \alpha}+\frac{\partial \boldsymbol{\delta}}{\partial \alpha}\right]+\mathbf{\Psi}^{*}(\alpha, t)\left[\frac{\partial \mathbf{I}^{*}}{\partial \mathbf{C}} \frac{\partial \mathbf{C}^{*}}{\partial \alpha}+\frac{\partial \mathbf{I}^{*}}{\partial \mathbf{K}} \frac{\partial \mathbf{K}^{*}}{\partial \alpha}+\frac{\partial \mathbf{I}^{*}}{\partial \alpha}-\frac{\partial \dot{\mathbf{K}}^{*}}{\partial \alpha}\right]\right. \\
& \left.+\frac{\partial \mathbf{\Psi}^{*}(\alpha, t)}{\partial \alpha}\left[\mathbf{I}\left(\mathbf{C}^{*}(\alpha, t), \mathbf{K}^{*}(\alpha, t), \alpha, t\right)-\dot{\mathbf{K}}^{*}(\alpha, t)\right]\right\} d \alpha \cdot e^{-\theta t} d t
\end{aligned}
$$

Since the equality constraint $\mathbf{I}(\cdot)=\dot{\mathbf{K}}(\cdot)$ always binds, the last term in the integrand of (19) vanishes. To simplify the formula, we integrate the term $\Psi^{*}(\alpha, t) \cdot \partial \dot{\mathbf{K}}^{*} / \partial \alpha \cdot d \alpha$ by parts to obtain

$$
\begin{aligned}
\int_{0}^{\infty}\left[\boldsymbol{\Psi}^{*}(\alpha, t) \frac{\partial \dot{\mathbf{K}}^{*}}{\partial \alpha} d \alpha\right] e^{-\theta t} d t= & -\int_{0}^{\infty}\left\{\left[\dot{\boldsymbol{\Psi}}^{*}(\alpha, t)-\theta \mathbf{\Psi}^{*}(\alpha, t)\right] \frac{\partial \mathbf{K}^{*}}{\partial \alpha} d \alpha\right\} e^{-\theta t} d t \\
& +\left.\left[\boldsymbol{\Psi}^{*}(\alpha, t) \frac{\partial \mathbf{K}^{*}}{\partial \alpha} d \alpha\right] e^{-\theta t}\right|_{0} ^{\infty} \\
= & \int_{0}^{\infty}\left[\mathbf{S}(\alpha, t) \frac{\partial \mathbf{K}^{*}}{\partial \alpha} d \alpha\right] e^{-\theta t} d t
\end{aligned}
$$

where $\mathbf{S}(\alpha, t)=\dot{\mathbf{\Psi}}(\alpha, t)-\theta \mathbf{\Psi}(\alpha, t)$ is the cost of holding capital during an infinitesimal period, $d t$. The second equality follows from the initial condition with $\mathbf{K}(0)$ fixed and the transversality condition $\lim _{t \rightarrow \infty}\left[\mathbf{\Psi}^{*}(\alpha, t) \partial \mathbf{K}^{*} / \partial \alpha\right] d \alpha e^{-\theta t}=0$. Using (20), we can rewrite (19) as

$$
\begin{aligned}
d W_{0}^{*}(\alpha)= & \int_{0}^{\infty}\left\{\frac{\partial U^{*}}{\partial \mathbf{C}}\left[\frac{\partial \mathbf{C}^{*}}{\partial \alpha}+\frac{\partial \boldsymbol{\delta}}{\partial \alpha}\right]+\mathbf{\Psi}^{*}(\alpha, t)\left[\frac{\partial \mathbf{I}^{*}}{\partial \mathbf{C}} \frac{\partial \mathbf{C}^{*}}{\partial \alpha}+\frac{\partial \mathbf{I}^{*}}{\partial \mathbf{K}} \frac{\partial \mathbf{K}^{*}}{\partial \alpha}+\frac{\partial \mathbf{I}^{*}}{\partial \alpha}\right]\right. \\
& \left.+\mathbf{S}^{*}(\alpha, t) \frac{\partial \mathbf{K}^{*}(\alpha, t)}{\partial \alpha}\right\} d \alpha \cdot e^{-\theta t} d t
\end{aligned}
$$

In fact, the expression in (21) can be greatly simplified by using the dynamic envelope theorem which eliminates all indirect effects. To fix ideas, however, we simplify the expression in two steps in order to better understand the rationale under the dynamic cost-benefit rule. First, we attempt to reduce the integral in (21) from an infinite time horizon to a finite time interval $[0, T]$, during which the project being carried out. Since the necessary conditions $\partial U^{*} / \partial \mathbf{C}+\mathbf{\Psi}^{*} \partial \mathbf{I}^{*} / \partial \mathbf{C}=0$ and $\mathbf{S}^{*}=\dot{\Psi}^{*}-\theta \mathbf{\Psi}^{*}=-\partial H^{*} / \partial \mathbf{K}$ 
hold along the conditional optimal trajectory with a given $\alpha=\alpha_{0}$, and the direct effects of an investment project are only confined within the project period $t \in[0, T]$ with $\partial \boldsymbol{\delta} / \partial \alpha=0$ and $\partial \mathbf{I}^{*} / \partial \alpha=0$ for all $t>T$, the part of the integral from time $T$ onwards would be zero. Thus, we can rewrite (21) as

$$
\begin{aligned}
d W_{0}^{*}(\alpha)= & \int_{0}^{T}\left[\frac{\partial U^{*}}{\partial \mathbf{C}}\left(\frac{\partial \mathbf{C}^{*}}{\partial \alpha}+\frac{\partial \boldsymbol{\delta}}{\partial \alpha}\right)+\mathbf{\Psi}^{*}(\alpha, t)\left(\frac{\partial \mathbf{I}^{*}}{\partial \mathbf{C}} \frac{\partial \mathbf{C}^{*}}{\partial \alpha}+\frac{\partial \mathbf{I}^{*}}{\partial \mathbf{K}} \frac{\partial \mathbf{K}^{*}}{\partial \alpha}+\frac{\partial \mathbf{I}^{*}}{\partial \alpha}\right)\right. \\
& \left.+\mathbf{S}^{*}(\alpha, t) \frac{\partial \mathbf{K}^{*}(\alpha, t)}{\partial \alpha}\right] d \alpha \cdot e^{-\theta t} d t \\
= & \int_{0}^{T}\left[\mathbf{P}^{*}(t) \Delta \mathbf{C}(t)+\mathbf{Q}^{*}(t) \Delta \mathbf{I}(t)+\mathbf{R}^{*}(t) \Delta \mathbf{K}(t)\right] \cdot \lambda(t) \cdot e^{-\theta t} d t
\end{aligned}
$$

\section{References}

Aronsson, T. Johansson, P.O. and Löfgren, K.G. (1997) Welfare Measurement, Green NNP and Sustainability: A Growth Theoretical Approach, Cheltenham: Edward Elgar.

Aronsson, T. and Löfgren, K.G. (1998) Green Accounting in Imperfect Market Economies, Environment and Resource Economics (1998 special issue on frontiers of Environmental and Resource Economics, 273-87).

Aronsson, T. and Löfgren, K.G. (1999) Welfare Equivalent NNP under Distributional Objectives, Economic Letters 63, 239-43.

Aronsson, T., Löfgren, K. G. and Backlund, K. (2004) Welfare Measurement in Imperfect Markets, Cheltenham: Edward Elgar.

Arrow, K., Dasgupta, P. and Mäler, K. G. (2003) Evaluating Projects and Assessing Sustainable Development in Imperfect Economies. Environmental and Resource Economics 26, 647-685.

Asheim, G.B. (2000) Green national accounting: why and how? Environment and Development Economics 5, 25-48.

Brock, W. A. (1977) A Polluted Golden Age, In (Smith, V., eds.) Economics of Natural and Environmental Resources, New York: Gordan and Breach.

Caputo, M.R. (1990) How to do Comparative Dynamics on the Back of an Envelope in Optimal Control Theory, Journal of Economic Dynamics and Control 14, 655-83. 
Dasgupta, P. (2001) Valuing Objects and Evaluating Policies in Imperfect Economies, The Economic Journal, 111(471), 1-29.

Dasgupta, P., Kriström, B. and Mäler, K. G. (1995) Current Issues in Resource Accounting. In (Johansson, P.O., Kriström, B. and Mäler, K.G., Eds.) Current Issues in Environmental Economics. Manchester University Press, Manchester and New York.

Dasgupta, P., Marglin, S. and Sen, A. (1972), Guidelines for project evaluation, New York: United Nations.

Dasgupta, P. and Mäler, K. G. (1997) The Resource Basis of Production and Consumption: An Economic Analysis. In: Dasgupta, P. and Mäler, K-G (eds) The Environment and Emerging Development Issues, Volume 1, Clarendon Press, Oxford, pp. 1-32.

Dasgupta, P. and K.G. Mäler (2000) Net National product, Wealth and Social WellBeing, Environment and Development Economics 5, 69-93.

Drèze, J. and Stern, N. H., (1987) The Theory of Cost-Benefit Analysis, in (Auerbach and Felstein, eds), Handbook of Public Economics (chapter 14).

Dixit, A., Hammond, P. Hoel, M. (1980) On Hartwick's Rule for Regular Maximin Paths of Capital Accumulation and Resource Depletion, Review of Economic Studies 47, 551-556.

Johansson, P.O., and Löfgren, K.G. (1996) On the Interpretation of Green NNPMeasures as Cost-Benefit Rules, Environment and Resource Economics 7, 243250.

Leonard, D. (1987) Co-state Variables Correctly Value Stocks at Each Instant: A Proof. Journal of Economic Dynamics and Control 11, 117-122.

Li, C. Z. and Löfgren, K. G.(2006) Comprehensive NNP, Social Welfare, and The Rate of Return. Economics Letters 90, 254-259.

Little, I. M. D., and Mirrlees, J. A. (1974) Project Appraisal and Planning for Development Countries: Social Cost-Benefit Analysis, Paris:OECD.

Seierstad, A., (1982) Differentiability Properties of the Optimal Value Function in Control Theory, Journal of Economic Dynamics and Control 4, 303-310

Weitzman, M L (1976) On the Welfare Significance of National Product in a Dynamic Economy, Quarterly Journal of Economics 90, 156-162

Weitzman, M.L. (2000) The Linearized Hamiltonian as Comprehensive NDP, Envi- 
ronment and Development Economics 5, 55-68.

Weitzman, M.L (2001) A Contribution to the Theory of Welfare Accounting, Scandinavian Journal of Economics 103, 1-24.

Weitzman, M.L. (2003) Income, Capital, and the Maximum Principle, Cambridge: Harvard University Press. 